\title{
Grußwort des Präsidenten
}

Liebe DMV-Mitglieder,

in der DMV-Geschäftsstelle hat es im Januar einen Wechsel gegeben. Frau Roswitha Jahnke, die seit August 2005 die Geschäftsstelle geleitet hatte, ist zum 12.1.2018 in den wohlverdienten Ruhestand gegangen. Vielen Dank an Frau Jahnke für viele Jahre hervorragender Arbeit und für ihren großartigen Einsatz für die DMV. Übernommen hat ihre Aufgaben ab dem 1.1.2018 Frau Andrea Kirstein-Gaekel, die schon ab August 2016 in der Geschäftsstelle Frau Jahnke zur Seite gestanden hatte. Ich wünsche Frau Kirstein-Gaekel einen guten Anfang bei den neuen Aufgaben und weiterhin viel Erfolg für die Zukunft.

Als neues Mitglied des Präsidiums der DMV möchte ich sehr herzlich Frau Ilka Agricola begrüßen. Frau Agricola wird sich unter anderem um die Nachwuchsförderung kümmern und damit eine der Aufgaben von Katrin Wendland übernehmen, deren Mitgliedschaft im Präsidium Ende 2017 ausgelaufen ist. Vielen Dank an Frau Wendland für ihr großes Engagement während ihrer Präsidiumszeit. Weiterhin Dank an Moritz Kaßmann und Wolfram Koepf, dass sie nach ihrer Wiederwahl für weitere vier Jahre im Präsidium mitarbeiten.

Auf der letzten Jahrestagung in Salzburg hat es auf Initiative von Herrn Hulek eine spezielle Sektion zur Bibliometrie gegeben. Vorstand und Präsidium der DMV haben dieses Thema aufgegriffen und auf ihren letzten Sitzungen diskutiert. Die Bewertung von Publikationsleistungen ist nicht nur ein zentraler Punkt in Berufungskommissionen, bei Forschungsförderungsanträgen und in Preiskomitees, sondern geht auch in Rankings von Universitäten und Fachbereichen ein. Außerdem gewinnt der Parameter „Publikationen“ bei internen Mittelverteilungsmodellen der Universitäten immer mehr an Bedeutung. Daher sehe ich es als unvermeidbar an, sich mit dem Thema Bibliometrie speziell vom Standpunkt der Mathematik aus zu befassen. Dazu gehören insbesondere auch Bewertung und Ranking von Zeitschriften, die schon in vielen anderen Ländern existieren und von Universitäten insbesondere bei Einstellung junger Mathematikerinnen und Mathematiker angewandt werden, und zwar orientiert an den leidigen „Impaktfaktoren“ (ein Beispiel hierfür ist China). Ich denke, hier sollte die Mathematik als Fach Stellung beziehen, nicht zuletzt, um formalen Einstufungen von Nicht-Fachleuten auf Verwaltungsebene zuvorzukommen.

Die DMV möchte hierzu die Diskussion unter ihren Mitgliedern anstoßen. Es wurden sieben Fragen zur Bibliometrie an einige Kollegen gesandt. Die Antworten finden Sie in dieser Ausgabe der Mitteilungen.

Die im letzten Grußwort erwähnte Sitzung der Kommission „Übergang Schule-Hochschule" hat wie angekündigt unter Beteiligung des Vorsitzenden der MNU, des 1 . Vorsitzenden der GDM und mir am 15. Dezember letzten Jahres stattgefunden. Es wurde beschlossen, je eine Arbeitsgruppe der drei Gesellschaften einzurichten, die einen gemeinsamen Forderungskatalog zur Verbesserung der Mathematikausbildung an den Schulen aufstellen sollen. Dieser soll dann auf einer Tagung zum Thema Mathematik an den Schulen, die in Berlin stattfinden soll, vorgestellt werden. Als ein möglicher Termin ist der 7. September 2018 ins Auge gefasst.

Auf Initiative der International Mathematical Union soll der 14. März (englisches Datum: $3.14=, \pi$-day“) der International Day of Mathematics werden. Ein entsprechender Antrag ist bei der UNESCO eingereicht, begleitet von Unterstützungsschreiben der mathematischen Fachgesellschaften aus aller Welt (auch eines der DMV).

Zum Abschluss möchte ich noch auf einige weitere wichtige Termine in diesem Jahr hinweisen. Die nächsten Gauß-Vorlesungen sind am 15.6.18 in Oldenburg und am 29.10.18 in Tübingen. Weiterhin finden zwei Tagungen statt, die gemeinsam mit mathematischen Gesellschaften anderer Länder organisiert werden, und zwar gemeinsam mit der Schweizerischen Mathematischen Gesellschaft vom 10.9. bis 13.9.18 in Genf und gemeinsam mit der Korean Mathematical Society vom 3.10. bis 6.10.18 in Seoul.

Mit den besten Grüßen Ihr Michael Röckner

verschiedener Einrichtungen und/oder Fakultäten geschrieben werden. Die unterschiedliche Struktur der einzelnen Universitäten macht einen Vergleich von Einzeleinrichtungen sehr aufwändig. Zu beachten ist auch, dass die inhärente Fehlerquote sowohl bei der Zuordnung von Referenzstrings zu Arbeiten als auch von Arbeiten zu Autoren und Institutionen sich bei den Gesamtzahlen zu einer Abweichung von bis zu $20 \%$ kumulieren kann. Die Jahre 2010 und 2014 wurden gewählt, da zum einen nach vier Jahren ein signifikanter Anteil der Zitationen relativ zum Publikationswachstum verfügbar ist (vgl. Abb. 4 auf S. 211), zum anderen für 2014 zumindest die Publikationsdaten einschließlich gedruckter Konferenzbände weitgehend vollständig erfasst sind (s. Anmerkung 2 auf Seite S. 213).

(Visualisierung: Christoph Eyrich. Umrisskarte: www.d-maps.com/m/europa/germany/allemagne/allemagne5o.pdf) 